\title{
Science of Synthesis: C-1 Building Blocks in Organic Synthesis, Workbench Edition, 2 Vol.
}

\author{
Edited by Piet W. N. M. van Leeuwen, $1^{\text {st }}$ ed., Thieme: Stuttgart, 2014, 1168 pp, \$ 549, ISBN: 9783131765116
}

The introduction of a one-carbon unit into an organic molecule through $\mathrm{C}-\mathrm{C}$ bond formation is a rather standard strategy for synthetic organic chemists. Dependent on the type of the C-1 reagent, nucleophilic or electrophilic substitution or addition reactions are traditionally used, and the reactions are usually categorized in a mechanistic way without special attention to the unique one-carbon reagent.

These two books provide a fresh and alternative look at the usual one-carbon building blocks and review how these can be applied to $\mathrm{C}-\mathrm{C}$ bond formation in practical organic synthesis, in both laboratory and industrial settings. The volume editor Piet van Leeuwen has a long career in industry and academia, and is thus the ideal person to lead this enterprise. Moreover, the editor is an expert in hydroformylation, a powerful synthetic reaction converting alkenes into saturated aldehydes with one extra carbon atom.

Van Leeuwen has gathered an impressive group of experts to review and discuss the use of the various C- 1 reagents such as carbon monoxide, carbon dioxide, diazomethane, hydrogen cyanide, nitromethane, formaldehyde, the several types of halogenated methane, methylene ylides, C-1 carbenes, and carbenoids.

The chapters are ordered according to the $\mathrm{C}-1$ reagent structure and the reaction type. In Volume 1, additions to alkenes, alkynes, and carbonyl compounds are treated. Examples are the earlier mentioned hydroformylation, cyclopropanation, the Michael addition, the Henry reaction, and cyanohydrin formation. Volume 2 discusses alkenations, cross-couplings, insertions, substitutions, and halomethylations. Here, the Wittig reaction and its modern variants, cross-couplings (e.g. with boron compounds), reactions of organometallic reagents with formaldehyde and carbon dioxide, the Mannich reaction, the Vilsmeier formylation, and the Kharasch reaction (radical addition of carbon tetrachloride) are reviewed.

Please note that this list of topics is not complete. There are many other reactions treated, including the carbonylation of alkynes, the hydrocyanation of alkenes, the cyanation of epoxides and aziridines, and mono-, di-, and trifluoromethylations.
Stereoselectivity receives appropriate attention, although the terminology is not so consistent. There is a chapter on asymmetric hydroformylation of alkenes, one on enantioselective conjugate addition of nitromethane (to electron-poor alkenes), and one on stereoselective addition of nitromethane (to $\mathrm{C}=\mathrm{O}$ and $\mathrm{C}=\mathrm{N}$ ). However, in all three chapters the main topic is enantioselectivity.

The industrial relevance of the chemistry receives casual attention in many chapters. One chapter (by Hans de Vries) is fully devoted to industrial applications of the hydroformylation of alkenes.

A valuable aspect of the Science of Synthesis book series is the principle that only really useful synthetic reactions are reviewed. Moreover, practical details are provided for the most representative procedures, including amounts of materials, conditions, yields, and safety issues.

Both volumes start with useful graphical abstracts of all chapters and a detailed table of contents, and end with an extensive subject and author index. Volume 1 commences with a somewhat wordy but interesting introduction by the editor on the philosophy behind the books, on the contents of the chapters, and on the choice of the chapter authors.

In summary, these two books contain very useful information for all scientists and practitioners of synthetic organic chemistry, ranging from $\mathrm{PhD}$ students working on synthetic methodology or total synthesis to process chemists in the drug industry. The books provide quick and insightful answers to research questions on introducing C-1 units into organic molecules. The unique collection of so many different reaction types in two books may even lead to cross-fertilization and stimulate further developments in the never ending science of synthesis.

Henk Hiemstra, Van 't Hoff Institute for Molecular Sciences, University of Amsterdam, The Netherlands 